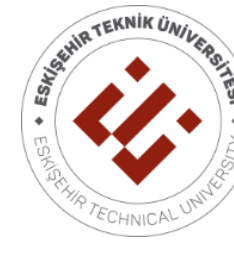

ESKISŞEHIR TECHNICAL UNIVERSITY JOURNAL OF SCIENCE AND TECHNOLOGY A- APPLIED SCIENCES AND ENGINEERING

\title{
ANALYSIS OF RESTRICTIONS INFLUENCE IN IMPLEMENTATION OF SPACE-TIME ACCESS METHODS
}

\author{
Mykola MOSKALETS ${ }^{1, *}$, Oleksiy SAMOYLENKOV ${ }^{1}$, Kyrylo KULISH ${ }^{1}$, \\ Kostiantyn SIELIVANOV ${ }^{1}$
}

${ }^{1}$ V.V. Popovskyy Department of Infocommunication Egineering, Kharkiv National University of Radio Electronics, Kharkiv, Ukraine

\begin{abstract}
The article analyzes the impact of restrictions on the effectiveness of space-time processing techniques in the antenna array during the implementation of space-time access methods. The method of qualitative and quantitative assessing the impact of the sum of the destabilizing factors in the form of restrictions affecting the signal to interference + noise ratio (SINR) is proposed on the basis of calculating the spatial coherence factor (SCF), which represents the value of the scalar product of the vectors of the desired signal and interference signal space.

The estimation of the upper and lower SINR values has been performed using spatial coherence factor in the assumption that the level of the interference signal interfering an SS at the entrance of AAA is significantly greater than the thermal noise power. The estimate of normalized index SINR reduction has been obtained regarding SCF.

The conditions of the SCF influence on the convergence rate of the adaptation process of the AAA control algorithms are shown and the analysis of the SCF boundary values is carried out, as a result of which the basic approaches to the construction of the AAA have been developed.
\end{abstract}

Keywords: Adaptive antenna array, Space-time processing

\section{INTRODUCTION}

Due to the severe lack of the radio frequency spectrum for supporting the ever-growing popularity of mobile systems (MSs), the need for the active implementation of space-time (ST) signal parameters has arisen. Among the active methods of such implementation are adaptive methods for space-time processing of received signals (STSP). The simplest and most obvious implementation of the method of organizing space-time access (STA) can be the implementation of an adaptive antenna array (AAA) at the base station, which implements the synthesis mechanism of a multipath direction pattern (DP) in the predetermined directions. Within each of the beam lobe, it is possible to receive SS signals localized in the allocated space. This scheme can be implemented using an adaptive antenna array.

The task of space-time access (STA) using adaptive antenna array (AAA) algorithms is essentially reduced to spatial selection of the signals from each of the subscriber stations (SSs) against the background of other SS and other emissions, interpreted as interference to the reception of signals of this station. An important advantage of these STA methods is that all other existing methods of multistation time-frequency access can work independently of STA. This allows the synthesis of STA methods and to analyze them independently of other methods. Moreover, the application of STA methods can be carried out already on the existing base and on existing wireless network technologies.

At the same time, when implementing STA, there are limitations associated mainly with the characteristics of AAA and the feasibility of adaptive STSP algorithms synthesized according to various criteria. The characteristics of AAA depend not only on the control algorithm, but also on the parameters of the antenna array itself: the number of antenna elements (AEs), directivity

*Corresponding Author: mykola.moskalets@nure.ua

Received: 13.10.209 Published:16.12.2019 
characteristics (DCs) and polarization characteristics of AEs, mutual influence of AEs, array configuration, etc. Moreover, all these factors in different degrees lead to a decrease in the realistically achievable signal-to-interference + noise ratio (SINR), and some may also cause a decrease in the rate of convergence of the adaptation process.

In this statement, the issues of analyzing various acting limiting factors of the STSP characteristics and assessment of its effectiveness taking into account the considered limitations are relevant for research.

\section{PURPOSE AND RESEARCH OBJECTIVES}

The aim of the study is to develop issues on the methodology for assessing the qualitative and quantitative influence of the sum of destabilizing factors used in STA technology, which reduces to the dependence of the SINR index on the level of interaction between the directing vector of the useful signal and the directing vectors of interfering signals.

To achieve this goal, the following tasks are carried out:

1) assessment of the space coherence factor ( $\mathrm{SCF}$ ) reduction, which is essentially the value of the scalar product of the useful signal vectors and the space of interfering signals;

2) assessment of the decrease degree in the relative SINR indicator in proportion to the SCF.

\section{MATERIALS AND METHODS FOR ANALYSIS OF AAA CHARACTERISTICS USING SPACE COHERENCE FACTOR}

The vector of the useful signal arriving along the main beam formed in the AAA is:

$$
\vec{X}_{c}(t)=a(t) e^{-\mathrm{j}\left(\omega_{0} t+\varphi(t)\right)} \vec{D}_{c},
$$

where $\vec{D}_{c}$ is the wavefront vector of the useful signal.

Similarly, we express the vector of the $v$ th interference in the form:

$$
\vec{X}_{n v}(t)=a_{v}(t) e^{-\mathrm{j}\left(\omega_{0} t+\varphi_{v}(t)\right)} \vec{D}_{n v},
$$

We assume that there is no coherence between the useful signal and the interference.

The correlation matrix of input signals can be written in the form [1]:

$$
R_{x x}=\sigma_{u i}^{2} I+P_{c} \vec{D}_{c}^{*} \vec{D}_{c}+\sum_{\gamma=1}^{L} P_{\gamma} \vec{D}_{m \gamma}^{+} \vec{D}_{n \gamma},
$$

where $\sigma_{u i}^{2}$ is the thermal noise power in the signal band;

$P_{c}$ is the power of the useful signal;

$P_{v}$ is the power of the $v$ th interference from a neighboring SS.

We represent the phase incursion $Z_{i}$ and $Z_{n v i}$, accordingly, in the form

$$
\begin{aligned}
& z_{i}=\vec{k}_{c} \cdot \vec{r}_{i}, \\
& z_{n v i}=\vec{k}_{n v} \cdot \vec{r}_{i}^{*},
\end{aligned}
$$


where $\vec{k}_{c}$ is the wavefront vector of the signal, $\vec{r}_{i}$ is the coordinate vector of the $i$ th AE.

When using the criterion of the minimum mean square deviation (MMSD), the optimal value of the weight vector (WV) will be determined by the expression

$$
\vec{W}_{o p t}=R_{x x}^{-1} \vec{R}_{x c}
$$

and for the output SINR, the expression [8] is true:

$$
\rho=P_{c} D_{c} R_{n n}^{-1} \vec{D}_{c}^{*} .
$$

Obviously, since the WV scaling does not change the SINR value, then expression (7) is also true for the criterion of the maximum signal-to-noise ratio (MSNR). Therefore, to reduce the calculations, let us consider of the MMSD criterion.

In the case when there is an influence of the 1st signal of the interfering SS (one interference), the expression $[2,3]$ is true for the optimal $\mathrm{WV}$ value:

$$
\vec{W}_{\text {opt }}=k\left\{\vec{D}_{c}-\frac{P_{1} \vec{D}_{c}^{+} \vec{D}_{1}}{\sigma_{u i}^{2}+P_{1} \vec{D}_{1} \vec{D}_{1}^{+}} \cdot \vec{D}_{1}\right\}
$$

where $k$ is the scalar coefficient.

Using the notation $\vec{D}_{i}^{+} \vec{D}_{j}=\delta_{i j}$, we obtain

$$
\vec{W}_{o p t}=\gamma\left\{\vec{D}_{c}-\frac{P_{1} \delta_{c 1}}{\sigma_{u i}^{2}+P_{1} \delta_{11}} \vec{D}_{1}\right\}
$$

The expression for the output SINR, taking into account expressions (7) and (9), is as follows:

$$
\rho=\frac{P_{c} \delta_{c c}}{\sigma_{u i}^{2}}\left\{1-\frac{P_{1}\left|\delta_{c 1}\right|^{2}}{\left(\sigma_{u i}^{2}+P_{1} \delta_{11}\right) \delta_{c c}}\right\}
$$

The value $\delta_{c 1}$ is the scalar product of the vectors $\vec{D}_{c}$ and $\vec{D}_{1}$. The absolute value of $\delta_{c 1}$ can be represented by the ratio:

$$
\left|\delta_{c 1}\right|=\left|\vec{D}_{1}\right|\left|\vec{D}_{c}\right| \cos \xi=\sqrt{\delta_{11}} \sqrt{\delta_{c c}} \cos \xi,
$$

where $\xi$ is the generalized angle between $\vec{D}_{c}$ and $\vec{D}_{1}$ in the complex vector space $\left|\vec{D}_{1}\right|=\sqrt{\vec{D}_{1}^{+} \vec{D}_{1}}=\sqrt{\delta_{11}} ;\left|\vec{D}_{c}\right|=\sqrt{\vec{D}_{c}^{+} \vec{D}_{c}}=\sqrt{\delta_{c c}}$.

Let us introduce the notation of the normalized spatial coherence factor (SCF) between the guide vectors of the signal $D_{c}$ and interference $D_{n}$.

Obviously, the SCF is uniquely determined through $\cos \xi$ :

$$
\gamma_{i j}=\frac{\vec{D}_{i} \vec{D}_{j}}{\left|\vec{D}_{i}\right|\left|\vec{D}_{j}\right|}=\frac{\delta_{i j}}{\sqrt{\delta_{i j}} \sqrt{\delta_{j j}}},
$$


where $i, j=c=1,2 \ldots \mathrm{n}$.

Then the expression for the SINR at the input can be represented as

$$
\rho=\frac{P_{\Sigma c}}{\sigma_{u i}^{2}}\left\{1-\frac{P_{n \Sigma}}{\sigma_{u i}^{2}+P_{n \Sigma}}\left|\gamma_{1 c}\right|^{2}\right\},
$$

where

$$
\begin{gathered}
P_{\Sigma c}=P_{c} \sum_{i=1}^{m}\left|A_{c}^{i}\right|^{2} \\
P_{n \Sigma}=P_{1} \sum_{i=1}^{m}\left|A_{n 1}^{i}\right|^{2}
\end{gathered}
$$

where $A_{c}^{i}$ and $A_{n 1}^{i}$ are determined respectively by the expressions:

$$
\begin{gathered}
A_{c}^{i}=\vec{E}_{i}\left(\theta, \Psi_{c}\right) \vec{\varepsilon}_{c} \\
A_{n v}^{i}=\vec{E}_{i}\left(\theta_{v}, \Psi_{v}\right) \vec{\varepsilon}_{v}
\end{gathered}
$$

where $\vec{E}_{i}\left(\theta, \Psi_{c}\right)$ is the vector directional characteristic of the $\mathrm{i}$ th $\mathrm{AE}$ according to the electric field intensity;

$\vec{\varepsilon}_{c}$ is the unit polarization vector of the incoming wave.

Let us estimate the lower and upper values of the SINR using expressions (13). Obviously, the maximum SINR value has the form

$$
\rho_{\max }=\frac{P_{\Sigma c}}{\sigma_{u i}^{2}} .
$$

When the interference is suppressed to zero and the SCF $\gamma_{1 c}=0$, the value of the SINR reaches the maximum. The maximum SINR does not depend on the location of the $\mathrm{AE}$ and the source of interference, but it is determined by the DCs of the AE and the polarization of the useful signal, as can be seen from expression (18). Under these conditions, the optimal WV has the form

$$
\vec{W}_{\text {opt }}=n \vec{D}_{c}^{+}
$$

Therefore, if AEs are identical and equally oriented, i.e. all $A_{c}^{i}$ are the same, then at the outputs of all AEs the useful signal is in phase and is coherently summed, as in a conventional phased antenna array (PAA). When $A_{c}^{i}$ are different, the SINR decreases despite the fact that the useful signal will still be coherently summed. The difference in values $A_{c}^{i}$ may be the result of environmental influences (masts, roofs, etc.).

To estimate the lower value of the SINR, we will assume that the level of the interference signal, i.e. of the interfering SS at the input of the AAA, is significantly more than thermal noise power

$$
P_{n \Sigma}>\sigma_{u i}^{2}
$$


then expression (13) is simplified and takes the form

$$
\rho=\frac{P_{\Sigma c}}{\sigma_{u i}^{2}}\left\{1-\left|\gamma_{1 c}\right|^{2}\right\}
$$

From the expression (21) it follows that the SINR at the output of the AAA depends only on $\rho_{\max }$ and $\left|\gamma_{1 s}\right|$ and does not depend on the interference power.

In addition, the lower value of the SINR is determined by expression (21). Using expressions (18), (21) we obtain

$$
\frac{P_{\Sigma c}}{\sigma_{u i}^{2}}\left\{1-\left|\gamma_{1 c}\right|^{2}\right\}<\rho \leq \frac{P_{\Sigma c}}{\sigma_{u i}^{2}}
$$

\section{RESULTS OF RESEARCH ON NORMALIZED INDICATOR OF SINR EFFICIENCY FROM SCF}

The dependences of the normalized SINR from on $\left|\gamma_{1 c}\right|$ are shown in Fig. 1. The normalized SINR value $\rho_{H}=\frac{\rho}{\rho_{\max }}$ varies within $\left[1 \div 1-\left|\gamma_{1 c}\right|^{2}\right]$.

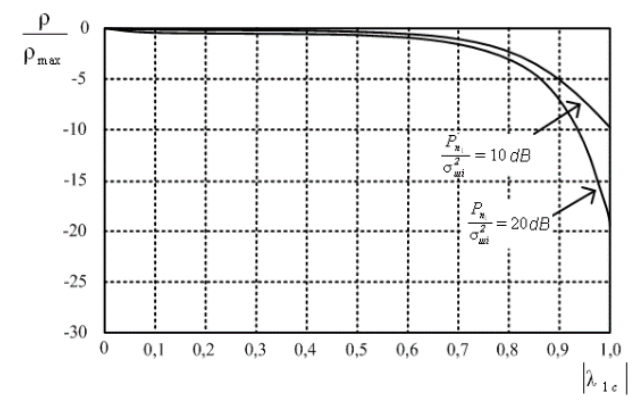

Figure 1. Schedule of reducing the dependence of SINR on the spatial coherence factor

As follows from the analysis of the dependences shown in Fig. 1, in the general case, for small values of $\frac{P_{n \Sigma}}{\sigma_{u i}^{2}}$, the SINR approaches the upper boundary, and for large values $\frac{P_{n \Sigma}}{\sigma_{u i}^{2}}$ it approaches the lower boundary.

However, at $\left|\gamma_{1 c}\right|<<1$, regardless of the value of the SINR $\frac{P_{n \Sigma}}{\sigma_{u i}^{2}}$, it always approaches the upper boundary. From this it follows that by decreasing the value of the SCF $\left|\gamma_{1 c}\right|$, it is possible to significantly improve the characteristics of AAA in the steady state.

The considered approach to assessing the characteristics of AAA based on the use of SCFs can be used in the presence of two or more interference [2]. Under these conditions, the generalized angle between $\vec{D}_{c}$ and the hyperplane formed by the vectors $\vec{D}_{n 1}, \vec{D}_{n 2}, \ldots, \vec{D}_{n n}$ represents the SCF. 
It should be noted that in the general case, SCFs depend on the following factors:

- angle of the useful signal and interference arrival;

- orientation and location of the $\mathrm{AE}$ in the array;

- AE directivity characteristics;

- polarization of the useful signal and interference.

To reduce the SCF, when designing AEs, it is necessary to properly select the array configuration, type and AE orientation. It is important to note that SCFs affect not only the output SINR, but in some cases they also affect the convergence rate of the adaptation process of AAA control algorithms synchronized on the basis on the gradient-based method [3,4]. This is caused by the fact that the convergence rate of gradient-based algorithms depends on the conditionality of the correlation matrix of the input signals. The conditionality of the correlation matrix, in turn, depends on the SCF.

In the case when a useful signal and one powerful interference affect the input of an $N$-element AAA, it can be shown that the eigenvalues of the correlation matrix can be determined $[3,4]$ :

$$
\left.\begin{array}{l}
\beta_{1}=\sigma_{u i}^{2}+\frac{1}{2}\left(P_{\Sigma c}+P_{\Sigma n}\right)+\frac{1}{2} \sqrt{\left(P_{\Sigma c}-P_{n \Sigma}\right)^{2}+4 P_{\Sigma c} P_{n \Sigma}\left|\gamma_{1 c}\right|^{2}} \\
\beta_{2}=\sigma_{u i}^{2}+\frac{1}{2}\left(P_{\Sigma c}+P_{\Sigma n}\right)-\frac{1}{2} \sqrt{\left(P_{\Sigma c}-P_{\Sigma n}\right)^{2}+4 P_{\Sigma c} P_{\Sigma n}\left|\gamma_{1 c}\right|^{2}} \\
\beta_{i}=\sigma_{u i}^{2}, i=3, \ldots, N
\end{array}\right\} .
$$

Then if $\left|\gamma_{1 c}\right|=1$, we have

$$
\left.\begin{array}{l}
\beta_{1}=\sigma_{u i}^{2}+P_{\Sigma c}+P_{\Sigma n} \\
\beta_{2}=\sigma_{u i}^{2}
\end{array}\right\}
$$

However, when $\left|\gamma_{1 c}\right|=0$, we get

$$
\left.\begin{array}{l}
\beta_{1}=\sigma_{u i}^{2}+P_{\Sigma n} \\
\beta_{2}=\sigma_{u i}^{2}+P_{c}
\end{array}\right\}
$$

Therefore, when $P_{c} \gg>\sigma_{u i}^{2}$, the convergence rate of the adaptation process at $\left|\gamma_{1 c}\right|=0$ is much higher than at $\left|\gamma_{1 c}\right|=1$, when

$$
\vec{D}_{1}=s \vec{D}_{c}
$$

where $s$ is the complex scalar.

In turn, (24) is satisfied when the following conditions are met [4]: 


$$
\begin{aligned}
& \left|A_{c}^{i}\right|=s_{1}\left|A_{n 1}^{i}\right|, \\
& \left(\operatorname{arctg}\left(\frac{\operatorname{Im}\left\{A_{c}^{i}\right\}}{\operatorname{Re}\left\{A_{c}^{i}\right\}}\right)-\operatorname{arctg}\left(\frac{\operatorname{Im}\left\{A_{n 1}^{i}\right\}}{\operatorname{Re}\left\{A_{n 1}^{i}\right\}}\right)-\left(\vec{k}_{c}-\vec{k}_{n 1}\right) r_{i}=2 \pi s_{2}\right\} \\
& i=1, \ldots N
\end{aligned}
$$

where $s_{1}$ and $s_{2}$ are real number constants.

From the analysis of the presented conditions (25) it follows [5] that:

1. For all AEs, the relationship between the signal gain and the interference should be the same.

2. The phase difference between the signal and the interference, as well as the phase differences between $A_{c}^{i}$ and $A_{n 1}^{i}$, conditioned by the location of the element, must be constant with accuracy up to $2 \pi$ (requirement for the plane of the phase front of the useful signal and interference).

\section{DISCUSSION OF RESEARCH RESULTS}

When $\left|\gamma_{1 c}\right|=1$, the described situation is presented in the form of AAA consisting of two isotropic and non-interacting AEs, separated in space by $d=\frac{\lambda_{c}}{2}$ with the angles of the signal and the interference arrival relatively to the normal to the line of the AE location, respectively $\theta_{c}=-90^{\circ}, \theta_{n}=90^{\circ}$.

Let us also assume that the polarization of the electromagnetic waves of the signal and the interference is the same. The AAA can either maximize or suppress interference, but at the same time it cannot achieve this simultaneously.

Under $\left|\gamma_{1 c}\right|=0$, when the inter-element distance $d=\frac{\lambda_{c}}{4}$, the array is able to simultaneously maximize the useful signal and completely suppress the interference.

If conditions (25) are not satisfied, it is easy to show that at $d=\frac{\lambda_{c}}{2}\left|\gamma_{1 c}\right|=0,8$ and in this case, the AAA can completely suppress the interference and partially useful signal. Through the use of different types of AEs, and due to the different orientations of identical elements, various directivity characteristics of AEs can be obtained. In the case of a priori accurate information on the angles of signals and interference arrival, the AAA could be constructed in such a way as to virtually eliminate the case of $\left|\gamma_{1 c}\right|=1$. However, there is no such information, and when constructing an array, it is necessary to aim for a situation where $\left|\gamma_{i c}\right|=1, i=1,2, \ldots, N$ would be small enough for a wide sector of interference arrival angles.

After analyzing these scenarios, the following approaches to the construction of AAA are proposed:

- use of irregular arrangement of the $\mathrm{AE}$ in the array (non-equidistant array);

- use of AEs with different DCs;

- use of different orientations of identical AEs.

The first approach makes it possible for AAA to carry out polarization-time processing of signals, i.e. distinguish between signals and interference by their polarization.

The last two approaches will inevitably reduce the potentially achievable SINR, but at the same time, this decrease in the maximum SINR is quite acceptable, since this will significantly reduce the likelihood of situations in which $\left|\gamma_{i c}\right|=1$. 


\section{REFERENCES}

[1] Monzingo RA, Miller TU. Adaptivnye antennye reshetki. Vvedenie v teoriyu. Moscow, 1986. 448 p.

[2] Balanis KA, Ioanides PI. Vvedenie v smart-antenny. Moscow, 2012. 200 s.

[3] Marchuk L.A. Prostranstvenno-vremennaia obrabotka syhnalov v lynyiakh radyosviazy / L.A. Marchuk. L., VAS, 1991. 136 s.

[4] Heng-Cheng Lin. Correction to "Spatial Correlations in Adaptive Arrays".IEEE Transactions on Antennas and Propagation.Year: 1982, Volume: 30, Issue: 6 Pages: 1268 - 1268, DOI: 10.1109/TAP.1982.1142929 Referenced in: IEEE Journals \& Magazines .

[5] Moskalets, N.V. Analyz vlyianyia ohranychenyi pry realyzatsyy metodov prostranstvennovremennoho dostupa. Visnyk natsionalnoho tekhnichnoho univer-sytetu "KhPI". 2016. №50 (122). S.95-100. 